\title{
Assistência ao pré-natal: participação do pai na gestação saudável
}

\author{
Prenatal assistance: father's participation in healthy pregnancy \\ Asistencia prenatal: participación del padre en un embarazo saludable
}

Recebido: 19/12/2020 | Revisado: 26/12/2020 | Aceito: 03/01/2021 | Publicado: 05/01/2021

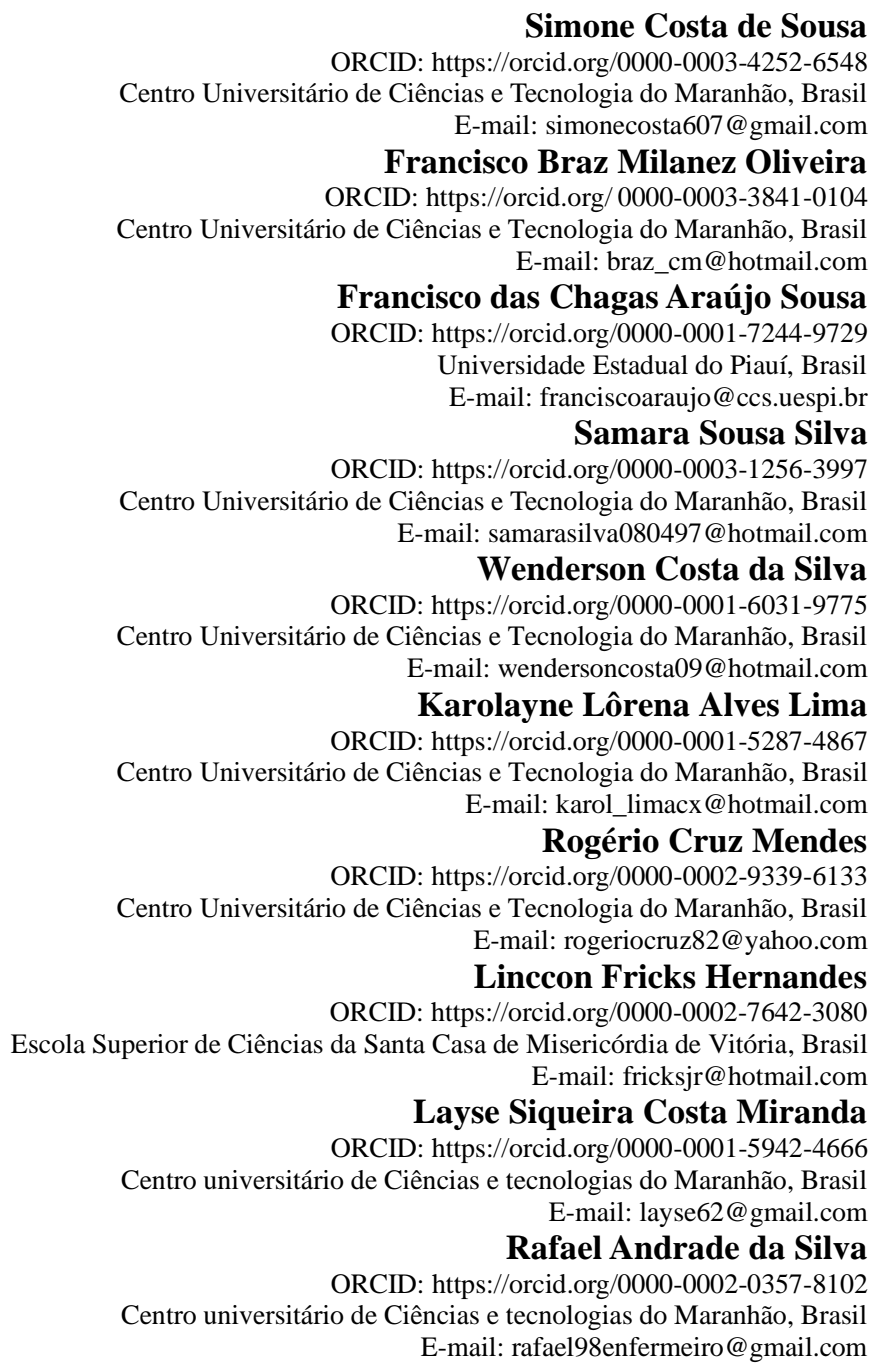

\section{Resumo}

A participação do pai é crucial para a rotina e desenvolvimento de uma gestação saudável, pois ambos se atentam a desenvolver atividades que beneficiam a gravidez. São inúmeros os benefícios associados ao envolvimento do pai na gestação, que devem ser incorporados desde o seu diagnóstico. Nesse contexto, este estudo teve como objetivo identificar quais os benefícios da participação do pai nas consultas de pré-natal. Trata-se de uma pesquisa bibliográfica do tipo revisão integrativa da literatura. Esse tipo de estudo foi escolhido por possibilitar a síntese e análise do conhecimento científico já produzido sobre o tema "ASSISTÊNCIA AO PRÉ-NATAL: participação do pai na gestação saudável". Consultou-se as bases de dados PubMed da National Library of Medicine, BVS (Biblioteca virtual da saúde) e CINAHL (Cumulatetive Index to Nursing and Allied Health Literature). Dos 10 (dez) estudos incluídos nesta revisão, seis (60\%) estavam na língua inglesa e quatro (40\%) na língua Portuguesa. Em relação ao ano de publicação, os artigos apresentaram variações, ganhando destaque o ano de 2017 (30\%). O Brasil foi o país com mais estudos incluídos (40\%), seguido da Holanda (20\%). O nível de evidência predominante foi composto principalmente por estudos transversais $(70 \%)$. Os dez estudos apresentaram dentro das práticas encontradas, os inúmeros benefícios da presença do parceiro frente às consultas de pré-natal, bem como maior igualdade de gênero, desenvolvimento saudável da criança, mais confiança da gestante e assistência à saúde paterna.

Palavras-chave: Paternidade; Pré-natal; Assistência de enfermagem. 


\begin{abstract}
The father's participation is crucial for the routine and development of a healthy pregnancy, as both are concerned with developing activities that benefit the pregnancy. There are countless benefits associated with the father's involvement in pregnancy, which must be incorporated since his diagnosis. In this context, this study aimed to identify the benefits of the father's participation in prenatal consultations. This is an integrative literature review. This type of study was chosen because it allows the synthesis and analysis of the scientific knowledge already produced on the topic "PRENATAL CARE: participation of the father in healthy pregnancy". The PubMed databases of the National Library of Medicine, VHL (Virtual Health Library) and CINAHL (Cumulative Index to Nursing and Allied Health Literature) were consulted. Of the 10 (ten) studies included in this review, six (60\%) were in the English language and four $(40 \%)$ in the Portuguese language. In relation to the year of publication, the articles presented variations, gaining prominence in 2017 (30\%). Brazil was the country with the most studies included (40\%), followed by the Netherlands (20\%). The predominant level of evidence was composed mainly of cross-sectional studies (70\%). The ten studies presented within the practices found, the numerous benefits of the partner's presence in the face of prenatal consultations, as well as greater gender equality, healthy child development, more confidence of the pregnant woman and paternal health care.
\end{abstract}

Keywords: Paternity; Prenatal; Nursing assistance.

\title{
Resumen
}

La participación del padre es fundamental para la rutina y el desarrollo de un embarazo saludable, ya que ambos se preocupan por desarrollar actividades que beneficien el embarazo. Son innumerables los beneficios asociados a la participación del padre en el embarazo, que deben incorporarse desde su diagnóstico. En este contexto, este estudio tuvo como objetivo identificar los beneficios de la participación del padre en las consultas prenatales. Ésta es una revisión integradora de la literatura. Se eligió este tipo de estudio porque permite la síntesis y análisis del conocimiento científico ya producido sobre el tema "CUIDADO PRENATAL: participación del padre en un embarazo saludable". Se consultaron las bases de datos PubMed de la Biblioteca Nacional de Medicina, VHL (Virtual Health Library) y CINAHL (Cumulative Index to Nursing and Allied Health Literature). De los 10 (diez) estudios incluidos en esta revisión, seis $(60 \%)$ estaban en inglés y cuatro (40\%) en portugués. En relación al año de publicación, los artículos presentaron variaciones, ganando protagonismo en 2017 (30\%). Brasil fue el país con más estudios incluidos (40\%), seguido de Holanda (20\%). El nivel de evidencia predominante estuvo compuesto principalmente por estudios transversales $(70 \%)$. Los diez estudios presentaron dentro de las prácticas encontradas, los numerosos beneficios de la presencia de la pareja frente a las consultas prenatales, así como una mayor equidad de género, desarrollo infantil saludable, más confianza de la gestante y atención de la salud paterna.

Palabras clave: Paternidad; Prenatal; Cuidado de enfermera.

\section{Introdução}

O período pré-natal é uma época de preparação física e psicológica para o parto, paternidade e maternidade, diante disso, é um momento de intenso aprendizado e uma oportunidade para os profissionais da equipe de saúde desenvolverem a educação como dimensão do processo de cuidar (Rios \& Vieira, 2007).

Atualmente, a definição do termo paternidade tem sofrido modificações, consequência das transformações que a sociedade vem sofrendo, seja no âmbito, cultural, econômico, científico e/ou político, construindo, assim, uma masculinidade contemporânea. O seu conceito também está relacionado a estigmas culturais, religiosos e familiares, pois esses fatores conceituam como o homem vivencia o momento, dessa forma, instalando um novo paradigma de como ser homem e como ser mulher (Rêgo, Souza, Rocha, \& Alves, 2016).

A forma como o homem se inclui durante as consultas de pré-natal manifesta como será a relação futura e envolvimento no ciclo gravídico-puerperal. Essa participação nas consultas de pré-natal fortalece o vínculo no momento do parto, pois influência em todas as fases que a gestante passa, sabendo-se que há uma necessidade muito grande, da gestante e do feto, de uma relação com amor e segurança. O companheiro, ao participar das consultas de pré-natal, torna possível maior vínculo e revigora os cuidados com a saúde da mulher (Caldeira, Ayres, Oliveira, \& Henriques, 2017).

A gestação é uma passagem para a parentalidade e cobra dos futuros pais adaptações biológicas e psicológicas a fins de preparação para o papel que devem adotar. De maneira biológica, a mulher é a responsável em carregar o novo ser, isso pode acontecer de maneira desigual, já que é um evento exclusivo da mulher, contudo é necessário desmitificar o ato de gestar como uma tarefa somente da mulher, posto se tratar de uma responsabilidade do casal. O fato de o homem não conter as 
mudanças corporais influencia no seu relacionamento, que pode acontecer mais devagar, e, em certas situações, somente após o nascimento (Cardoso, Silva Junior, Bomatti, Santos, \& Ribeiro, 2018).

São inúmeros os benefícios associados ao envolvimento do pai na gestação, que devem ser incorporados desde o diagnóstico da gravidez, de modo que a consulta de pré-natal seja a primeira a oferecer uma grande oportunidade para esse envolvimento, cabendo ressaltar que mulheres que têm esse apoio apresentam menos complicações durante o trabalho de parto e puerpério, bem como sintomas físicos e emocionais (Cardoso et al., 2018).

Os serviços de saúde estão preparados para atender somente a mulher gestante, o que dificulta o acesso do homem. É bastante difundido que a presença da figura do pai é importante, mas ela ainda é desvalorizada pelos profissionais de saúde, o que pode ser explicado pela falta de acolhimento e infraestrutura precárias. Outra condição é o fato de o homem ter mais dificuldade em perceber suas necessidades de saúde, rejeitando a possibilidade de adoecer. O pré-natal não pode ser visto só como um momento de alterações fisiológicas, mas também de alterações emocionais (Henz, Medeiros, \& Salvadori, 2017).

A relevância deste estudo justifica-se por abordar os principais entraves da população masculina ao acompanhamento de suas parceiras durante o pré-natal, quais os impactos trazem para a gestante, seus benefícios e como a enfermagem pode intervir nesse processo, posto que a equipe de enfermagem desempenha papel muito importante desde o início e pode ser uma fonte muito importante para o preparo e incentivo das intervenções.

Há uma grande necessidade em criar estratégias e intervenções para a participação do pai na gestação, pois ainda há um distanciamento dos serviços de saúde por parte dos homens, que impossibilita o contato precoce deste. Assim os resultados deste estudo poderão contribuir para apontar quais dificuldades ainda são perceptíveis para o público masculino em relação à participação do homem durante as consultas de pré-natal.

Esse estudo teve como problemática: Qual a importância da presença do pai durante o pré-natal? Para tal objetivou-se identificar quais os benefícios da participação do pai nas consultas de pré-natal. E, especificamente, investigar quais fatores pode interferir na ausência do pai durante as consultas de pré-natal; discutir qual o período se manifesta os sentimentos de contribuição e apoio do parceiro da gestante e, por fim, descrever sentimentos adquiridos no envolvimento do casal durante a gestação.

\section{Metodologia}

Realizou-se uma pesquisa bibliográfica, do tipo revisão integrativa da literatura. Essa técnica foi satisfatória pois possibilitou a análise cientifica já realizada sobre a temática "ASSISTÊNCIA AO PRÉ-NATAL: participação do pai na gestação saudável”. Esta revisão utilizou a metodologia proposta no estudo de Oliveira et al. (2016).

A revisão integrativa de literatura é uma metodologia que tem como objetivo substanciar os resultados encontrados através da análise de forma sistematizada, organizada e ampla. Tem como característica principal abranger informações em um universo vasto sobre o assunto de interesse, conduzindo para a descrição de conceitos, revisão de teorias ou revisão da metodologia dos estudos. Sua metodologia fortalece a união entre os dados da literatura teórica e o senso comum, propiciando melhor entendimento acerca do tema de interesse (Ercole, Melo, \& Alcoforado, 2014).

O tema estabeleceu a estruturação da estratégia PICO, sendo caracterizada um acrônimo para Paciente (P), Interesse (I), Contexto (Co), em que foi disposto para a construção da questão norteadora desta pesquisa na literatura: Qual a importância do acompanhamento do pai durante o pré-natal?

Para a identificação de artigos que replicassem a questão norteadora, utilizou-se de descritores indexados e não indexados (palavras-chave) nos idiomas português, inglês e espanhol. Esses descritores foram obtidos através do Medical Subject Headings (MESH), dos Descritores em Ciências da Saúde (DeCS) e dos títulos CINAHL, caracterizado no Quadro 1. 
Examinou-se por meio de descritores e palavras-chave as bases de dados PubMed da National Library of Medicine; BVS (Biblioteca Virtual da Saúde), coordenada pela BIREME e composta de bases de dados bibliográficas produzidas pela Rede BVS, como LILACS, além da base de dados Medline e outros tipos de fontes de informação; e CINAHL (Cumulative Index to Nursing and Allied Health Literature). Os descritores foram combinados nos bancos de dados, por meio da busca eletrônica avançada booleana através do $A N D$ e $O R$

Quadro 1. Elementos da estratégia PICo, descritores e palavras-chave utilizados - Caxias, MA, Brasil, 2020.

\begin{tabular}{|c|c|c|c|c|c|}
\hline \multicolumn{2}{|c|}{ ELEMENTOS } & MESH & DECS & $\begin{array}{c}\text { TÍTULOS } \\
\text { CINAHL }\end{array}$ & $\begin{array}{c}\text { PALAVRAS- } \\
\text { CHAVE }\end{array}$ \\
\hline P & "Gestante" & "Pregnancy" & "Pregnant Women " & "Pregnancy" & "Pregnant Women" \\
& & & "Mujeres" & "Mujeres" \\
I & "Paternidade" & "Paternity" & "Embarazadas" & "Gestantes " \\
\hline Co & & & "Paternity" & "Paternity" & "Paternity" \\
& & & "Paternidad" & "Paternidade" \\
\hline
\end{tabular}

Fonte: Descritores, Títulos e Palavras-chaves (2020).

Nos critérios de inclusão e exclusão da pesquisa foi empregado estudos disponíveis em seu total publicados no limite temporal nos últimos cinco anos, de 2015 até 2020, nos idiomas Português, Espanhol e Inglês, que respondessem à questão norteadora. Foram excluídos textos duplicados, textos incompletos, buscas em livros, revistas, resumos, teses, relatos técnicos, monografias, bem como outras formas de publicações que não fossem artigos científicos.

A análise para escolha dos estudos foi realizada em dois períodos, a saber: no primeiro momento, os estudos foram pré-selecionados segundo os critérios de inclusão e exclusão e de acordo com a estratégia de funcionamento e busca de cada base de dados. Já o segundo momento, consiste na leitura minuciosa dos artigos selecionados de cada base, segundo os quesitos de inclusão e que respondiam os objetivos do estudo.

Encontrou-se na base PUBMED, como busca total foram encontrados 744 (setecentos e quarenta e quatro) artigos, aplicando na pesquisa os filtros que limita por texto completo dos últimos cinco anos com humanos, obtendo-se 55 (cinquenta e cinco) artigos, destes foram analisados títulos e resumos e teve como resultado final de 03 (três) artigos.

Encontrou-se 119 (centro e dezenove) estudos como busca geral na BVS, sendo que limitando a busca para artigos com texto completo realizado com humanos nos últimos cinco anos, obteve-se 30 (trinta) estudos, destes foram analisados títulos e resumos resultando 4 (quatro) estudos foram acrescentados a pesquisa.

Na base CINAHL, como busca total foram encontrados 1098 (mil e noventa e oito) estudos, aplicando na pesquisa o filtro que limita por texto completo dos últimos cinco anos com humanos, 152 (cento e cinquenta e dois) estudos, destes foram analisados títulos e resumos e teve como resultado final de 3 (três) artigos. 
As evidências científicas foram classificadas segundo os níveis propostos por Bork (2011), em que temos: Nível 1 Revisão sistemática; Nível 2 - Ensaios clínicos randomizados; Nível 3 - Estudo de Coorte; Nível 4 - Estudo de Caso Controle; Nível 5 - Séries de casos; Nível 6 - Opiniões de especialistas; e Nível 7 - Estudos pré-clínicos (animais/in vitro).

\section{Resultados}

Dos 10 (dez) estudos incluídos nesta revisão, seis (60\%) estavam na língua inglesa e quatro (40\%) na língua Portuguesa. Em relação ao ano de publicação, os artigos apresentaram variações, ganhando destaque o ano de 2017 (30\%). O Brasil foi o país com mais estudos incluídos (40\%), seguido da Holanda (20\%). O nível de evidência predominante foi composto principalmente por estudos transversais (70\%).

Tabela 1. Análise descritiva das produções científicas acerca da participação do pai para uma gestação saudável. Caxias, MA, Brasil, 2020.

\begin{tabular}{lcc}
\hline VARIÁVEIS & N & \% \\
\hline Abordagem do estudo & 06 & 60,0 \\
Quantitativo & 04 & 40,0 \\
Qualitativo & & \\
Delineamento da pesquisa & 07 & 70,0 \\
Estudo Transversal & 02 & 20,0 \\
Estudo de Coorte & 01 & 10,0 \\
Ensaio clínico aleatorizado controlado & & \\
Idioma & 06 & 60,0 \\
Inglês & 04 & 40,0 \\
Português & & \\
Nível de evidência & 01 & 10,0 \\
Dois & 02 & 20,0 \\
Três & 07 & 70,0 \\
Seis & & \\
Procedência & 01 & 10,0 \\
Alemanha & 04 & 40,0 \\
Brasil & 01 & 10,0 \\
China & 01 & 10,0 \\
Estados Unidos & 02 & 20,0 \\
Holanda & 01 & 10,0 \\
Inglaterra & & \\
Distribuição temporal & & 10,0 \\
2015 & 01 & 20,0 \\
2016 & 02 & 30,0 \\
2017 & 03 & 20,0 \\
2018 & 02 & 10,0 \\
2019 & 01 & 10,0 \\
2020 & &
\end{tabular}

Fonte: Pesquisa direta (2020).

A prevalência dos estudos abordava os principais efeitos da participação do parceiro durante o pré-natal, bem como, demonstrando o perfil da gestante, perfil do parceiro e principais limitações atreladas durante esse processo.

Em relação ao perfil da gestante, nos achados têm-se uma prevalência de gestantes com idade igual ou superior a 18 anos, com período mínimo de 20 semanas e máximo de 28 semanas gestacionais (Bonifácio, 2018; Kortsmit et al., 2020; Caldeira et al., 2017; Gobel et al., 2019; Cardoso et al., 2018; De Cock et al, 2016; Lucassen et al., 2016). 
Entre os achados do perfil do parceiro da gestante, notou-se ter idade igual ou superior a 18 anos, ser parceiro fixo, acompanhou/participou das consultas de pré-natal (Bonifácio, 2018; Kortsmit et al., 2020; Henz et al., 2017; De Cock et al, 2016; Dheensa, Metcalfe, \& Williams, 2015; Lucassen et al., 2016).

Em relação às principais limitações dos estudos, observou-se que companheiros que não tinham acesso a um telefone móvel (Bonifácio, 2018); oferta de horário incompatível com o atendimento (Henz et al., 2017); escassez de assunto sobre o tema, pouco conteúdo em relação a experiência paterna (Gobel et al., 2019); e ao fato de não existir nenhum instrumento para avaliar o vínculo no pré e pós-natal (De Cock et al., 2016).

\section{Análise dos benefícios do acompanhamento do parceiro no pré-natal}

Nove estudos abordaram os benefícios do parceiro/pai frente as consultas de pré-natal. Em relação aos benefícios todos os estudos mostraram bons resultados ao vínculo precoce do parceiro no pré-natal (Bonifácio, 2018; Kortsmit et al., 2020; Chan, Emery, Fulu, Tolman, \& Ip, 2017; Caldeira et al., 2017; Gobel et al., 2019; Cardoso et al., 2018; Henz et al., 2017; De Cock et al, 2016; Dheensa et al., 2015).

Bonifácio (2018), Henz et al., (2017), Chan et al. (2017) e Dheensa et al. (2015), revelam que levar o parceiro da gestante mais próximo dos serviços de saúde está associado positivamente com a saúde paterna. O vínculo do pai nas consultas de pré-natal deve-se à melhoria do nascimento e resultados de desenvolvimento, psicológicos e cognitivos da criança (Kortsmit et al., 2020; Chan et al., 2017; Henz et al., 2017; De Cock et al, 2016).

Também se observou que a participação do parceiro reflete maior igualdade de gênero e mais proteção da família (Henz et al., 2017; Chan et al., 2017). Além de proporcionar aproximação precoce da tríade mãe-pai-filho (Caldeira et al., 2017; Gobel et al., 2019; Cardoso et al., 2018; De Cock et al, 2016), e favorecer o apoio e cuidado com a saúde da mulher (Caldeira et al., 2017; Dheensa et al., 2015). Além disso, o pré-natal gera uma oportunidade para sanar as dúvidas que surgem sobre os cuidados com o bebê nos primeiros dias de vida e preparo para o nascimento (Caldeira et al., 2017; Henz et al., 2017).

\section{Análise dos fatores que podem interferir na ausência do pai durante as consultas de pré-natal}

Acerca dos achados sobre os fatores que podem interferir na ausência do pai nas consultas de pré-natal, identificou-se que a falta de apoio dos profissionais/família foi o mais predominante, cerca de $50 \%$ dos estudos abordaram esse fator (Kortsmit et al., 2020; Caldeira et al., 2017; Cardoso et al., 2018; Henz et al., 2017; Dheensa et al., 2015), o horário desfavorável das consultas foi outro fator evidenciado como influenciador na ausência (Bonifácio, 2018; Caldeira et al., 2017; Cardoso et al., 2018; Henz et al., 2017).

Em relação aos fatores como a falta de infraestrutura local para atender às consultas e parceiro de baixa renda ou escolaridade apresentaram um número expressivo de achados, (Bonifácio, 2018; Caldeira et al., 2017; Gobel et al., 2019; Henz et al., 2017).

Quanto a não identificação do parceiro da gestante nos serviços de saúde, maus-tratos sofridos pelo casal em relação ao atendimento, ausência de capacitações dos profissionais e hostilidade do parceiro, ou seja, grosseria e não aceitação em participar das consultas, foram os fatores com o menor número de achados (Bonifácio, 2018).

\section{Análise dos sentimentos adquiridos no envolvimento do casal durante a gestação}

Dentre os achados a presença do parceiro incentivou o aumento da busca de conhecimentos das mulheres sobre o cuidado nesse período (Bonifácio, 2018). 
Um dos conhecimentos desenvolvidos durante a gestação foi facilitar a transição para a paternidade para mães e pais (Kortsmit et al., 2020). O parceiro demonstrou maior senso de maturidade, responsabilidade, companheirismo e compartilhamento durante a gravidez (Chan et al., 2017; Caldeira et al., 2017).

Alguns sentimentos foram relatados quando o homem é excluído ou retirado desse contexto ocorrendo o aparecimento de ciúmes, ansiedade e solidão como consequência (Caldeira et al., 2017). Em relação aos achados onde o parceiro apresentase ansioso, encontrou somente essa ansiedade relacionada à espera de seu primeiro filho ou quando é uma gestação complicada (Henz et al., 2017; Dheensa et al., 2015). Pais com problemas de saúde mental podem perceber o feto como uma fonte adicional de angústia ou irritação (Gobel et al., 2019; De Cock et al., 2016).

Há mais confiança das gestantes em seus parceiros nos momentos de dificuldade e fortalecimento dos laços familiares (Cardoso et al., 2018; Henz et al., 2017). Os achados caracterizam o suporte emocional, calor e consideração positiva sejam características maternas, enquanto os pais tendem a ser mais físicos e estimulantes (Lucassen et al., 2016).

\section{Discussão}

\section{Benefícios da participação do pai nas consultas de pré-natal}

Os achados relacionados à caracterização dos estudos quanto aos benefícios da participação do pai/parceiro nas consultas de pré-natal revelaram que ele representa um personagem fundamental durante a gestação, corroborando com os estudos de Mello, Parauta, Saldanha, e Lemos (2020), Medeiros et al. (2020), Cavalcanti e Holanda (2019), Trindade, Cortez, Dornelas e Santos (2019), Menezes e Scorsolini-Comin (2019), Queiroz et al. (2019), Santos, Ferreira e Freitas (2018), Amaro (2018) e Magalhães, Couto, Fernandes e Oliveira (2018) afirmam que a presença paterna como integrante da rede de apoio à mulher na gestação, parto e puerpério, proporciona o estabelecimento precoce do vínculo entre pai e bebê/criança e desenvolvimento do bem-estar da mulher.

De acordo com os achados sobre a participação do parceiro com a gestante nas rotinas de pré-natal, notou-se melhora no desenvolvimento cognitivo e mental da criança, de modo a corroborar com os estudos de Bustamante (2019), Redshaw e Henderson (2013) e Menezes e Scorsolini-Comin (2019) apresentam as mesmas características.

Os estudos destacam que a paternidade reflete maior igualdade de gênero, em concordância com os estudos de Menezes e Scorsolini-comin (2019), Trindade et al., (2019) e Bustamante (2019) que comentam as mudanças nos papéis femininos, que, por sua vez, acarretaram alterações dos masculinos, ampliando repercussões no exercício da parentalidade e nos novos arranjos familiares.

Em relação aos estudos que apontam o pré-natal como um período crucial para a saúde paterna, corroborando com Amaro (2018) e Santos et al. (2018) afirma-se que, ao captar o público masculino precocemente, dando destaque na promoção e realização de testes rápidos e exames, acrescenta-se à assistência a possibilidade de rastrear possíveis comorbidades no sujeito, além de oferecer suporte psicológico, entendendo que este momento é permeado por sentimentos ambíguos e dúvidas.

As pesquisas sugerem que as consultas de pré-natal constituem momentos propícios para sanar as dúvidas referentes aos primeiros cuidados com o bebê e preparo para o nascimento, corroborando com os estudos de Balica e Aguiar (2019) e Queiroz et al. (2019), que afirmam que o período gestacional é um momento de buscar atendimento especializado para o prénatal e de se preparar para o parto.

\section{Fatores que podem interferir na ausência do pai durante as consultas de pré-natal}

Os achados relacionados aos fatores que podem interferir na ausência do parceiro nas consultas de pré-natal relatam que a ausência de apoio dos profissionais e/ou da família foi o fator que mais se destacou nos estudos dessa revisão, divergindo com as evidências de outros estudos, como nos realizados por Medeiros et al. (2020), Trindade et al. (2019), Nass et al. (2017) 
e Moreira, Gomes, Ribeiro (2016) que comentam que os profissionais ocupam um papel estratégico na conquista da presença dos homens, no apoio às decisões relativas ao seu cuidado e de quem com eles convive.

O horário desfavorável das consultas de pré-natal foi destacado como um dos fatores que dificultam o acompanhamento do pai, em contraste a isto, no aporte teórico de Brasil (2019) destaca o horário de funcionamento estendido em diversas UBS, favorecendo assim a continuidade do atendimento.

No que diz respeito à falta de capacitação e treinamento dos profissionais quanto à prestação de serviços à saúde do homem, corroborando com o estudo de

Araujo, Azevedo, Magalhães, Ramos e De Jesus, (2017), que afirmam que existe escassez de projetos para qualificação dos profissionais que compõem a equipe para lidarem com o público masculino.

Os achados correspondentes à falta de identificação dos parceiros nas consultas de pré-natal, corroborando com Queiroz et al. (2019) em seu estudo, destacam a baixa adesão dos parceiros no acompanhamento da parceira durante o prénatal, e um dos fatores contribuintes é falta de interesse e conhecimento acerca da importância nesse período.

De acordo com os achados, a baixa renda ou escolaridade dos parceiros apresentaram-se como fatores responsáveis pelo desinteresse do parceiro nas consultas de pré-natal, corroborando com o estudo de Santos et al. (2018) destacam, ainda, que há certa resistência pela procura aos serviços, principalmente os de atenção primária à saúde e nas camadas populares mais baixas, em que os traços culturais são marcantes e o acesso às informações em saúde é limitado.

Em relação à falta de infraestrutura, local para atender às consultas, corroborando com os estudos de Pompermaier e Freitas (2020), comentam sobre as inúmeras dificuldades enfrentadas pelos homens quanto à participação das consultas do prénatal. Os espaços de saúde, quase que exclusivamente, são de atenção voltada à saúde da mulher e da criança, não havendo espaços para os homens, fazendo-os sentir que não fazem parte desse ambiente. A falta de infraestrutura local para atender às consultas é um dos fatores destacado, no estudo de Oliveira et al. (2017), abordam a importância da infraestrutura local para dá continuidade à assistência, bem como, proporcionar atendimento humanizado a gestante e familiares.

\section{Período que se manifestam os sentimentos de contribuição e apoio do parceiro da gestante}

É durante as consultas de pré-natal o parceiro se identifica mais e nota sua contribuição e apoio, porém nos estudos de estudo de Lima, Cazola e Pícoli (2017), estar junto da mulher durante a amamentação é a maneira que os pais encontraram para favorecer sua participação, logo é fundamental para o sucesso desse processo. Ainda, afirmam Rêgo et al. (2016), que o pai se tornou um aliado na amamentação.

De acordo com os estudos analisados, o parceiro se envolve de maneira expressiva após o nascimento da criança, pois a materialização é um fator determinante para o vínculo, corroborando com Bustamante (2019), em seu estudo realizado no Brasil, discorre que a participação paterna após o nascimento da criança é um período considerado crucial para o desenvolvimento, que exige grandes demandas de cuidado, gera efeitos nos adultos cuidadores e nas relações entre eles, em que modos de se relacionar começam a ser construídos. Ainda, afirmam Miura, Guimarães, Lima, Santos e Ferro (2019) que durante o período da gestação o homem tem dificuldades em viver sua paternidade porque não sente a presença física do bebê.

De acordo com os achados, o parceiro demonstra esse apoio durante toda a gestação, corroborando com os estudos de Santos et al. (2018), citam que o homem contemporâneo percebe a paternidade desde a gravidez, iniciando ainda nessa fase a construção de vínculos afetivos.

Diante dos achados, o momento em que o parceiro mais contribui é durante o trabalho de parto, corroborando com o estudo Leite (2018), que discorre que o apoio afetivo e a participação ativa do parceiro atua de forma positiva sobre a gestante durante o parto, sendo responsável pela diminuição da queixa e sensação de dor, uso de analgésico, números de cesarianas, além de tempo de internação pós-parto. 


\section{Sentimentos adquiridos no envolvimento do casal durante a gestação}

De acordo com os estudos analisados, a gestante desenvolve mais confiança no seu parceiro, corroborando com Medeiros et al. (2020), que, em seu estudo realizado no Pará, afirmam que há mais confiança das gestantes em seus parceiros nos momentos de dificuldade, persistindo o fortalecimento dos laços familiares. Em contrapartida, Silva, Gabriel, Cherer e Piccinini (2017) faz menção da importância que o pai seja percebido não só como fonte de apoio à mãe, mas que se leve também em consideração suas próprias necessidades, angústias, dificuldades perante a paternidade e do relacionamento com o filho.

Dentre os estudos, a presença do parceiro incentivou o aumento do interesse das mulheres sobre o cuidado nesse período, corroborando com o estudo de Dal-Rosso et al. (2019), afirmam ser possível perceber que a paternidade produziu transformações positivas nas suas parceiras, como o cuidado de si e a busca por informações pertinentes durante esse período.

Corroborando com o estudo de Quitete e Monteiro (2018), destacam que envolvimento dos pais traz uma mistura de sentimentos não apenas para os homens, mas, principalmente, para as mulheres, que se sentem mais seguras e preparadas para esse momento especial. A participação do pai fornece apoio emocional, ajuda as mulheres a suportar a dor e a tensão do parto. E a presença de alguém de sua confiança faz com que se sintam mais satisfeitas, confiantes e felizes.

Em relação ao pai/parceiro mostrar-se ansioso, Silva e Martins (2018) explicam esse fenômeno em decorrência das mudanças hormonais que moldam a sua adaptação ao papel paterno.

As pesquisas abordaram uma melhor transição para paternidade, ou seja, uma mudança mais efetiva de mães e pais, corroborando com o estudo de Magalhães et al. (2018), realizado em Portugal, que comentam que a inclusão do pai durante a gravidez desempenha um papel fundamental, aumentando a confiança e conduzindo a uma boa mudança para a parentalidade de todos os membros da família.

Os estudos caracterizam que o suporte emocional, o calor e consideração positiva sejam características maternas, enquanto os pais tendem a ser mais físicos e estimulantes, corroborando com o estudo de Moreira et al. (2016), afirmam que a mulher é a mais passiva em desacordo com o homem que se apresenta com mais pressa e estimulante, sendo mais de difícil de lidar. Rêgo et al. (2016) confirmam os achados em seu estudo, que o parceiro vem apresentando novas funções, companheirismo, cuidador, protetor e mais responsabilidade.

\section{Conclusão}

A partir da avaliação da participação do pai para uma gestação saudável, identificou-se os inúmeros benefícios da presença do parceiro frente às consultas de pré-natal, bem como maior igualdade de gênero, desenvolvimento saudável da criança, mais confiança da gestante e assistência à saúde paterna. Diversos são os fatores que influenciam a ausência do parceiro nas consultas de pré-natal, a ausência de apoio dos profissionais e/ou da família ainda permeiam a realidade, demonstrando assim a fragilidade do atendimento. As consultas de pré-natal ainda são a melhor forma de fortalecimento da paternidade, representando um momento importante e cheio de mudanças físicas e emocionais. Considera-se, ainda, que a gestação é uma experiência desafiadora e repleta de sentimentos, na qual a mulher e sua família precisam passar de forma saudável.

Como limitações, notou-se escassez de estudos na área, bem como evidências de baixo nível que abordavam a questão norteadora. Há também maior necessidade de revisão ou mesmo reformulação da assistência ao pré-natal, com ênfase na qualidade da assistência, para desenvolvimento de uma gestação saudável. É necessária a busca de estratégias diferenciadas para alcançar os grupos familiares socialmente mais vulneráveis.

O próximo passo, diante do estudo realizado, é validar a assistência na atenção básica, ou seja, nas unidades básicas de saúde, onde são rotineiramente realizados os pré-natais de acordo com o protocolo realizado pelos profissionais de 
enfermagem, a fim de ampliar o atendimento às gestantes e familiares no intuito de aumentar o interesse da participação do parceiro.

Reforça-se, ainda, que os resultados desta pesquisa podem resultar em inovações nos atendimentos já desenvolvidos no serviço de saúde, que podem refletir sobre o tema, propondo a inclusão do homem/pai em um pré-natal que ultrapasse as questões biológicas e inclua espaços para o esclarecimento de dúvidas e potencialização do cuidado. Espera-se que mais estudos sejam realizados nessa linha de investigação, com novas formas de abordar a temática, visando diminuir as barreiras que impedem a participação do pai no período do pré-natal.

\section{Referências}

Amaro, N. C. S. (2018). Valorização da paternidade no pré-natal: revisão narrativa de literatura (Trabalho de Conclusão de Curso). Universidade Federal de Campina Grande, Brasil.

Araujo, A. D. F., Azevedo, A. M. F., Magalhães, G. M. O., Ramos, L. V. A., \& De Jesus, C. S. (2017). Pré-natal masculino: a inclusão na saúde do homem. Revista de Trabalhos Acadêmicos UNIVERSO, 2(3), 29-46.

Balica, L. O., \& Aguiar, R. S. (2019). Percepções paternas no acompanhamento do pré-natal. Rev. Aten. Saúde, 17(61), 114-126.

Bonifácio, L. P. (2018). Pré-natal do parceiro: uso da estratégia PRENACEL para melhorar o envolvimento masculino no pré-natal (Tese de Doutorado). Universidade de São Paulo, Brasil.

Bork, A. M. T. (2011). Enfermagem baseada em evidências. Guanabara Koogan.

Botelho, L. L., Cunha, C. C. A., \& Macedo, M.O. (2011). Método da Revisão Integrativa nos estudos Organizacionais. Gestão e Sociedade, 5(11), 121-136.

Brasil. (2019). Instrutivo de Adesão ao Programa Saúde na Hora. Ministério da Saúde.

Bustamante, V. (2019). Participação paterna no cuidado durante o primeiro ano de vida. Pensando familias, 23(1), 89-104.

Caldeira, L. Á., Ayres, L. F. A., Oliveira, L. V.A., \& Henriques, B. D. (2017). A visão das gestantes acerca da participação do homem no processo gestacional. Revista de Enfermagem do Centro-Oeste Mineiro, 7, e1417.

Cardoso, V. E. P. S., Silva Junior, A. J., Bonatti, A. F., Santos, G. W. S., \& Ribeiro, T. A. N. (2018). A Participação do Parceiro na Rotina Pré-Natal Sob a Perspectiva da Mulher Gestante. Revista de Pesquisa: Cuidado é Fundamental Online, 10(3), 856-862.

Cavalcanti, T. R. L., \& De Holanda, V. R. (2019). Participação paterna no ciclo gravídico-puerperal e seus efeitos sob a saúde da mulher. Enfermagem em Foco, 10(1), 93-98.

Chan, K. L., Emery, C. R., Fulu, E., Tolman, R. M., \& Ip, P. (2017). Association Among Father Involvement, Partner Violence, and Paternal Health: UN Multi-Country Cross-Sectional Study on Men and Violence. American Journal of Preventive Medicine, 52(5), 671-679.

Dal-Rosso, G. R., Silva, S. O., Pieszak, G. M. Ebling, S. B. D., \& Silveira, V. N. (2019). Experiências narradas por homens no exercício da paternidade: rompendo paradigmas. Rev. Enferm. UFSM, 9, 1-18.

De Cock, E. S. A., Henrichs, J., Vreeswijk, C. M. J. M., Maas, A. J. B. M., Rijk, C. H. A. M., \& van Bakel, H. J. A. (2016). Continuous feelings of love? The parental bond from pregnancy to toddlerhood. Journal of Family Psychology, 30(1), 125-134.

Dheensa, S., Metcalfe, P. A., \& Williams, R. (2015). O que os homens querem da triagem pré-natal? Resultados de um estudo de entrevista na Inglaterra. Obstetrícia, 31(1), 208-214.

Dos Santos, E. M., \& Ferreira, V. B. (2016). Pré-natal masculino: significados para homens que irão (re)experienciar a paternidade. Revista Funec Científica, Multidisciplinar, 5(7), 62-78.

Ercole, F. F., Melo, L. S., \& Alcoforado, C. L. G. (2014). Integrative review versus systematic review. Revista Mineira de Enfermagem, 18(1), 1-2.

Göbel, A., Barkmann, C., Arck, P., Hecher, K., Schulte-Markwort, M., Diemert, A., \& Mudra, S. (2019). Couples' prenatal bonding to the fetus and the association with one's own and partner's emotional well-being and adult romantic attachment style. Midwifery, $79,102549$.

Henz, G. S., Medeiros, C. R. G., \& Salvadori, M. (2017). A inclusão paterna durante o pré-natal. Rev Enferm Atenção Saúde, 6(1), 52-66.

Kortsmit, K., Garfield, C., Smith, R. A., Boulet, S., Simon, C., Pazol, K., \& Warner, L. (2020). Paternal Involvement and Maternal Perinatal Behaviors: Pregnancy Risk Assessment Monitoring System, 2012-2015. Public Health Reports, 135(2), 253-261.

Leite, D. A. (2018). Vivências do pai no pré-natal, pré-parto e parturição no século XXI (Trabalho de Conclusão de Curso). Universidade Federal de Uberlândia, Uberlândia, Brasil.

Lima, J. P., Cazola, L. H. O., \& Pícoli, R. P. (2017). Participação do pai no processo de amamentação. Cogitare enferm, 1(22), 01-07. 
Lucassen, N., Tiemeier, H., Luijk, M. P. C. M., Linting, M., Bakermans-Kranenburg, M. J., Van IJzendoorn, M. H., \& Lambregtse-Van den Berg, M. P. (2016). Expressed Emotion During Pregnancy Predicts Observed Sensitivity of Mothers and Fathers in Early Childhood. Parenting, 15(3), 158-165.

Magalhães, J., Couto, G., Fernandes, C., Oliveira, I. (2018). Envolvimento do pai no período prénatal: revisão integrativa. Revista de Investigação \& Inovação em Saúde, 1(2), 53-65.

Medeiros, T. S. P., Arruda, L. Y., Moia, M. Y. S., Machado, E. P., Igreja, P. N., Silva, K. C, \& Silva, S. M. (2020). Percepção das gestantes sobre a participação familiar no pré-natal. Braz. J. of Develop, 6(4), 18777- 18792.

Mello, M. G., Parauta, T. C., Saldanha, B. L., \& Lemos, A. (2020). The young father involvement in the prenatal care: the perspective of health professional. Revista de Pesquisa Cuidado É Fundamental Online, 12, 95-100.

Menezes, M. S. L., \& Scorsolini-Comin, F. (2019). Envolvimento paterno na relação mãe-bebê: revisão integrativa da literatura. Psicol. rev., 25(1), 19-39.

Miura, P. O., Guimarães, M. R., Lima, E. F. O., Santos, K. A. M. S., \& Ferro, M. E. S. S. (2019). A participação do pai no pré-natal: experiência de companheiros de adolescentes grávidas. Gepnews, 2(2), 299-303.

Moreira, M. C. N., Gomes, R., \& Ribeiro, C. R. (2016). E agora o homem vem?! Estratégias de atenção à saúde dos homens. Cadernos de Saúde Pública, 32(4), e00060015.

Nass, E. M. A., Lopes, M. C. L., Alves, B. D., Marcolino, E., Serafim, D., Higarashi, I. H., \& Marcon, S. S. (2017). Vivências da maternidade e paternidade na adolescência. Revista Baiana de Enfermagem, 31(2), e16629.

Oliveira, F. B. M., Costa, A. C. A. L.; Alves, D. L., F. J. F., Macedo, M. S., Santos, R. D. (2016). Relação entre a sobrecarga de trabalho e erros de administração de medicação na assistência hospitalar. Revista Ciências \& Saberes, 2(2), 325-334.

Oliveira, A. C., Xavier, A. V. P., Silva, A. L., Escorcio, D. S. R., Rodrigues, E. S., \& Sousa, G. R. A. (2017). As proporções do cuidado pre-natal na consulta de enfermagem. Revista Uningá, 54(1), 176-184.

Pompermaier, C., \& Freitas, G. T. (2020). A participação paterna no pré-natal. Anuário Pesquisa e Extensão Unoesc Xanxerê, 5 , e24268.

Queiroz, E. N. S., Santos, A. A., Melo, K. K. O., Magalhães, A. Y. F., Martins, L. L. F., Portela, F. B. S., \& Ferreira, V. P. (2019). Avaliação da adesão ao prénatal do parceiro: impacto no trinômio. Braz. J. Hea. Rev., 2(5), 4835-4841.

Quitete, J. B., \& Monteiro, J. A. M. B. (2018). A participação do pai no parto domiciliar planejado: um ato significativo para a mulher. Revista Enfermagem UERJ, 26, e18682.

Redshaw, M., \& Henderson, J. (2013). Fathers' engagement in pregnancy and childbirth: evidence from a national survey. BMC Pregnancy and Childbirth, $13(1), 1-15$.

Rêgo, R. M. V., Souza, Â. M. A., Rocha, T. N. A., \& Alves, M. D. S. (2016). Paternidade e amamentação: mediação da enfermeira. Acta Paulista de Enfermagem, 29(4), 374-380.

Rios, C. T. F., \& Vieira, N. F. C. (2007). Ações educativas no pré-natal: reflexão sobre a consulta de enfermagem como um espaço para educação em saúde. Ciência \& Saúde Coletiva, 12(2), 477-486.

Santos, D. S. S., Rosário, C. R., Brito, H. E. S., Soares, T. M., \& Bispo, T. C. F. (2018). A importância da participação paterna no pré-natal, para a compreensão do parto e puerpério. Revista Brasileira de Saúde Funcional REBRASF, 5(2), 55-68.

Santos, W. P., Ferreira, J. A., \& Freitas, F. B. D. (2018). As implicações do processo de paternidade para o comportamento masculino. Rev. APS, 21(2), 291299.

Silva, C. S. M., \& Martins, C. A. (2018). A perspectiva neuro-hormonal da paternidade: uma revisão. Anais do Congresso Internacional da Associação Portuguesa dos Enfermeiros Obstetras, Coimbra, Portugal, 5.

Silva, M. R., Gabriel, M. R., Cherer, E. Q., \& Piccinini, C. A. (2017). Os conceitos de envolvimento e experiência nos estudos sobre paternidade. Arquivos Brasileiros de Psicologia, 69(3), 116-132.

Trindade, Z., Cortez, M. B., Dornelas, K., \& Santos, M. (2019). Pais de primeira viagem: demanda por apoio e visibilidade. Saúde e Sociedade, 28(1), 250261. 